\title{
ZnTe-based UV sensors
}

\author{
S.Yu. Pavelets, Yu.N. Bobrenko, T.V. Semikina, G.I. Sheremetova, B.S. Atdaiev, \\ K.B. Krulikovska, M.A. Mazin \\ V. Lashkaryov Institute of Semiconductor Physics, NAS of Ukraine \\ 41, prospect Nauky, 03680 Kyiv, Ukraine \\ Tel.: 38 (044) 525-61-52; e-mail: pavelets@voliacable.com
}

\begin{abstract}
A $p$-ZnTe $/ n$-CdSe heterojunction was used for making polycrystalline ZnTebased UV sensors. The heteropair components have the same crystal structure and close lattice parameters. ZnTe and CdSe were grown using thermal evaporation and quasiclosed space condensation. A transparent current collecting electrode for the surfacebarrier structure of $p-\mathrm{ZnTe} / n$-CdSe heterojunction was made of degenerate $p-\mathrm{Cu}_{1.8} \mathrm{~S}$. Surface relief of ZnTe grown on different substrates was studied with scanning atomic force microscopy. The energy band offset diagrams of heterojunction are built and photosensitivity spectra are presented.
\end{abstract}

Keywords: zinc telluride, heterojunction, UV sensor, surface-barrier structure, energy band offset diagram, crystal structure.

Manuscript received 18.12.15; revised version received 05.04.16; accepted for publication 08.06.16; published online 06.07.16.

\section{Introduction}

The wide-gap II-VI semiconductor compounds are promising materials for making UV sensors. Among them are those based on the surface-barrier structures whose transparent component is degenerate copper sulfide $p$ $\mathrm{Cu}_{1.8} \mathrm{~S}$, while photosensitive components are $n$-type semiconductors $\mathrm{ZnS}$ [1], ZnSe [2] or CdS [3]. The most efficient are $p-\mathrm{Cu}_{1.8} \mathrm{~S} / n$-CdS UV sensors. Their high sensitivity is determined by two factors: (i) at $\mathrm{Cu}_{1.8} \mathrm{~S}$ film thicknesses of about $10 \mathrm{~nm}$, the conditions for maximal absorption of UV radiation by the surface-barrier structure used are realized in the region of sweeping electric field, and (ii) a considerable contribution to photocurrent from hot electrons generated in the $\mathrm{Cu}_{1.8} \mathrm{~S}$ layer by high-energy radiation [4].

Interest to $p$-ZnTe as a promising material for semiconductor photoelectronics is determined by both its wide gap $E_{g}=2.26 \mathrm{eV}$ (similarly to the well known GaP
UV photoelectric converters (PEC) [5] with $E_{g}=$ $2.25 \mathrm{eV}$ ) and possibility of making an abrupt $p$-ZnTe/ $n$-CdSe heterojunction. The same crystal structure of two semiconductors ( $\mathrm{ZnTe}$ and $\mathrm{CdSe}$ ) and a small mismatch of their lattice parameters (less than 1\%) are necessary conditions for making a high-quality heterojunction. In this work, we studied the $p$-ZnTe/n-CdSe heterojunction with a transparent current collecting electrode of degenerate $p$ - $\mathrm{Cu}_{1.8} \mathrm{~S}$.

\section{Fabrication of photoelectric converters}

The heterojunction components $\mathrm{ZnTe}$ and CdSe were grown using thermal evaporation and quasi-closed space condensation. Cadmium selenide (with the electron concentration $n \approx 10^{16} \mathrm{~cm}^{-3}$ ) was deposited onto Mo foil used as a lower current collecting electrode. ZnTe with the concentration of majority charge carriers (holes) $p=$ $(5 \ldots 8) \cdot 10^{14} \mathrm{~cm}^{-3}$ was grown on CdSe. The obtained 
structure was illuminated on the side of a transparent $p-\mathrm{Cu}_{1.8} \mathrm{~S}$ electrode that was deposited onto the $\mathrm{ZnTe}$ surface using vacuum sputtering.

It is known that structure perfection of heteroepitaxial layers as well as their physical properties depend largely on the density of defects appearing due to mismatch of lattice parameters of the connecting materials. For the ZnTe-CdSe pair, that mismatch lies within $0.16 \ldots 0.95 \%[6,7]$, so one would expect that rather good junctions can be obtained.

It should be noted that it is possible to get an ideal lattice matching for the considered pair by using interlayers of solid solutions with variable composition $[1,2]$. In this work, we used interlayers of $(\mathrm{ZnTe})_{x}(\mathrm{CdSe})_{1-x}$ solid solutions. The layers of $\mathrm{CdSe},(\mathrm{ZnTe})_{x}(\mathrm{CdSe})_{1-x}$ of variable composition and $\mathrm{ZnTe}$ were grown sequentially on the Mo substrate in a single technological cycle from two independent sources (zinc and cadmium chalcogenides) by using quasi-closed space condensation. We studied structural features of polycrystalline ZnTe layers obtained on different substrates: Mo foil, $\mathrm{Mo} / \mathrm{CdSe}$ and $\mathrm{Mo} / \mathrm{CdSe} /(\mathrm{ZnTe})_{x}(\mathrm{CdSe})_{1-x}$.

The surface relief was studied using the scanning atomic force microscopy (AFM) with a NanoScope IIIa Dimension 3000 system. The 3D height maps of typical $10 \times 10 \mu \mathrm{m}$ surface fragments were obtained in the periodic probe-surface contacting mode. The AFM patterns of surface fragments for $\mathrm{ZnTe}$ samples grown on different substrates are shown in Fig. 1. The ZnTe film surfaces are typical for polycrystalline layers: they are close-packed grain arrays with clear faceting.

Both grain size and faceting essentially depend on the substrate type. To illustrate, a ZnTe film obtained on Mo foil (Fig. 1a) has grains of the smallest characteristic diameter (within 2 to $5 \mu \mathrm{m}$ ) as compared with other films, and those grain faces have the biggest angle of inclination to the horizontal (about $30^{\circ}$ ). Small grains and big inclination angles may indicate a considerable level of stresses and defects.

If $\mathrm{ZnTe}$ is grown on the $\mathrm{Mo} / \mathrm{CdSe} /(\mathrm{ZnTe})_{x}(\mathrm{CdSe})_{1-x}$ substrate (Fig. 1b), then grain lateral dimensions are $5 \ldots 8 \mu \mathrm{m}$, and there appears a clear trend to form crystallites with faces oriented in the substrate plane (angle up to $10^{\circ}$ ). Also, attention is drawn to the presence of a number of growth terraces on the face surfaces as well as availability of small as well as very big grains. One may assume that the film has not reach equilibrium state under the given growth conditions.

If $\mathrm{ZnTe}$ is grown on the $\mathrm{Mo} / \mathrm{CdSe}$ substrate (i.e., directly on the CdSe surface), then the processes of grain coagulation and coalescence are accelerated. As a result, the characteristic grain size increases considerably (to $5 \ldots 10 \mu \mathrm{m})$ and the averaged angle of inclination to the substrate surface decreases to $10 \ldots 15^{\circ}$ (Fig. 1c). Under the given growth conditions, the crystal structure is formed in the conditions of equilibrium. This is indicated by presence of big smooth faces and a considerable number of $120^{\circ}$ grain boundary junctions.

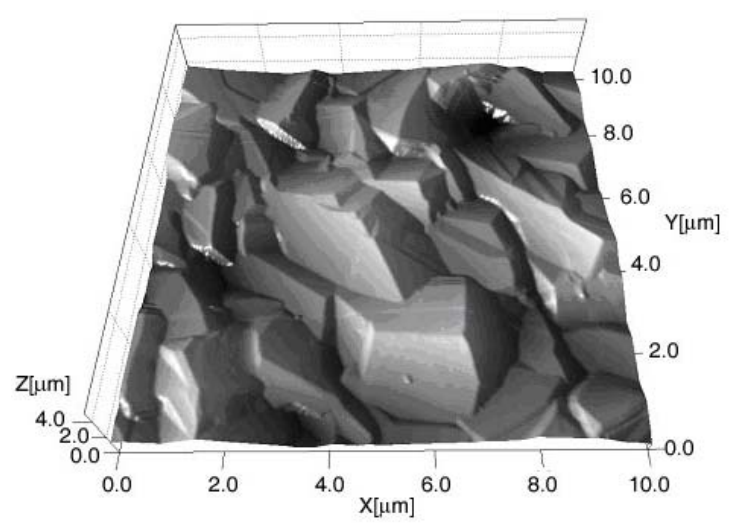

a)

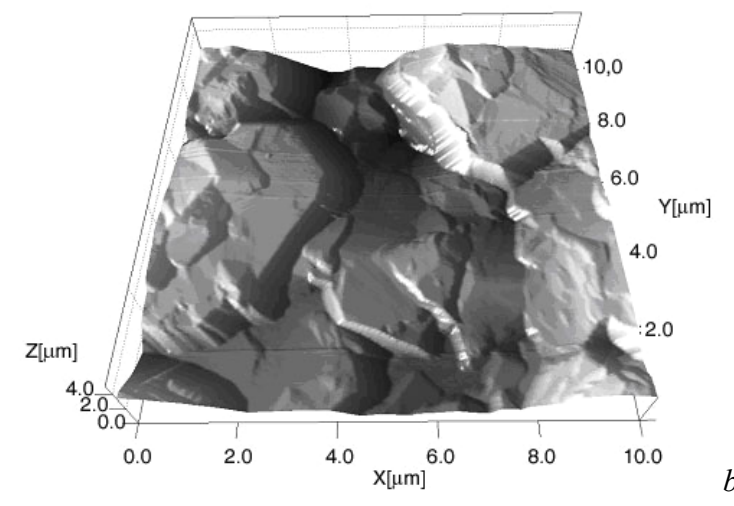

b)

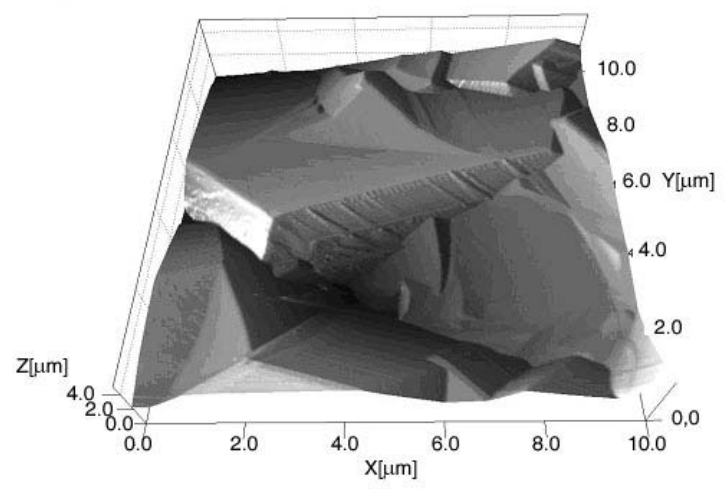

c)

Fig. 1. AFM patterns of surface fragments for $\mathrm{ZnTe}$ samples grown on different substrates: (a) - Mo foil; (b) $\mathrm{Mo} / \mathrm{CdSe} /(\mathrm{ZnTe})_{x}(\mathrm{CdSe})_{1-x} ;(\mathrm{c})-\mathrm{Mo} / \mathrm{CdSe}$.

Thus, the result obtained for heterostructure with $(\mathrm{ZnTe})_{x}(\mathrm{CdSe})_{1-x}$ interlayer indicates that in such a case conditions for epitaxial growing the ZnTe layers with a perfect structure have not improved. Indeed, probability of $\mathrm{ZnTe}+\mathrm{CdSe} \leftrightarrow \mathrm{ZnSe}+\mathrm{CdTe}$ exchange reaction seems to lead to formation of materials $(\mathrm{ZnSe}, \mathrm{CdTe})$ with drastically differing lattice parameters and appearance of structural defects at the initial stage of ZnTe layer growth on the $\mathrm{Mo} / \mathrm{CdSe} /(\mathrm{ZnTe})_{x}(\mathrm{CdSe})_{1-x}$ substrate. 


\section{Energy band offset diagrams of heterostructures and photocurrent spectra of switching diode photoelectric converters}

One cannot understand the physical processes in semiconductor devices without knowing energy band diagrams. This statement becomes most evident when considering the features of heterojunctions. The principal item for abrupt heterojunctions is determination of relative position of conduction and valence bands on each side of interface, i.e., determination of sign and value of conduction and valence band offsets, $\Delta E_{c}$ and $\Delta E_{v}$.

For some time, the Anderson model [8] (known as the electron affinity rule) remained generally accepted when determining energy band offsets. In 1975, however, Kroemer drew attention to the fact that the above rule is unreliable. Even if having reliable data on the electron affinity value, still application of the electron affinity rule will depend on unobvious approximations made at determination of the interfaces between two semiconductors and much more abrupt semiconductor-vacuum interface.

Let us use the data obtained in [9] to build energy band offset diagram for the $p-\mathrm{ZnTe} / n-\mathrm{CdSe}$ heterojunction. The authors of [9] followed the procedure used in photoemission core level spectroscopy and determined the valence band offsets $\Delta E_{\mathrm{v}}$ (separation between the valence band maxima of two semiconductor compounds forming a heterostructure) for III-V and II-VI compounds. The valence band offset calculated for ZnTe and CdSe according to [9] was $\Delta E_{v}=0.66 \mathrm{eV}$. Partial overlapping the energy bands is realized for the above pair of semiconductors (Fig. 2a), so the conduction band offset can be calculated as

$\Delta E_{\mathrm{c}}=E_{g 1}+\Delta E_{v}-E_{g 2}=1.22 \mathrm{eV}$

where $E_{g 1}=2.26 \mathrm{eV}$ and $E_{g 2}=1.7 \mathrm{eV}$ are the band gap widths of $\mathrm{ZnTe}$ and $\mathrm{CdSe}$, respectively.

To plot a quantitative energy band offset diagram for the heterojunctions obtained, one should, along with knowing the energy band offsets, estimate the Fermi level positions in $\mathrm{ZnTe}$ and $\mathrm{CdSe}$ (the absolute energy values $F_{1}$ and $F_{2}$, respectively). The concentration of majority charge carriers (holes) in the used ZnTe layers was $p \approx 5 \cdot 10^{14} \mathrm{~cm}^{-3}$; it corresponds to $F_{1} \approx 0.28 \mathrm{eV}$. For CdSe, respectively, the electron concentration was $n \approx$ $3 \cdot 10^{16} \mathrm{~cm}^{-3}$, which corresponds to $F_{2}=0.1 \mathrm{eV}$.

The total contact potential difference $e U_{d}$ specified by the difference in thermodynamic work functions is $e U_{d 1}+e U_{d 2}$; here $e U_{d 1}\left(e U_{d 2}\right)$ is the potential difference in $\mathrm{ZnTe}(\mathrm{CdSe})$ in the state of equilibrium. It is easy to verify (see Fig. 2a) that

$e U_{d}=\left(E_{\mathrm{g} 1}-F_{1}\right)-\left(\Delta E_{c}+F_{2}\right)$,

for the heterojunctions studied, $e U_{d}=(0.66 \ldots 0.7) \mathrm{eV}$. This value agrees well with the maximal photo-e.m.f. values that were observed for PEC studied at white light.

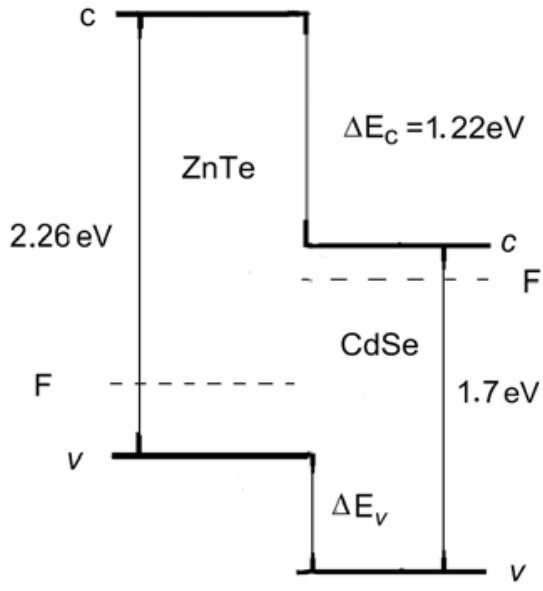

a)

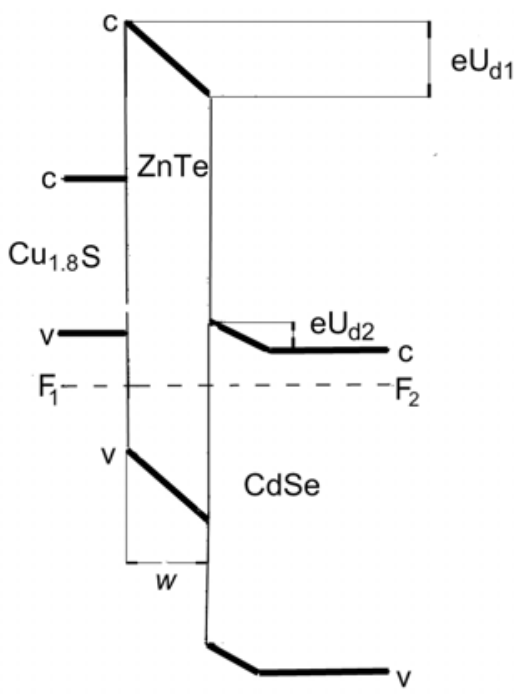

b)

Fig. 2. Energy band offset diagrams for: (a) - separated ZnTe and $\mathrm{CdSe} ;(\mathrm{b})-p-\mathrm{Cu}_{1.8} \mathrm{~S} / p-\mathrm{ZnTe} / n-\mathrm{CdSe}$ heterostructure $(F-$ Fermi level, $E_{c}-$ conduction band, $E_{v}-$ valence band).

Shown in Fig. $2 \mathrm{~b}$ is the energy band offset diagram for $p-\mathrm{Cu}_{1.8} \mathrm{~S} / p-\mathrm{ZnTe} / n-\mathrm{CdSe}$ PEC. The UV PEC design is optimal: $d \leq w$, where $d$ is the thickness of the main part (ZnTe layer) of photoactive structure body and $w-$ space charge region (SCR) width in ZnTe. PEC is illuminated from the side of a transparent current collecting electrode (degenerate $\mathrm{Cu}_{1.8} \mathrm{~S}$ ), the thickness of which is about $10 \mathrm{~nm}$. Thus, the investigated structures belong to the surface-barrier ones whose maximal absorption of UV radiation occurs in the region of sweeping electric field.

Fig. 3 shows the photocurrent spectra for two PEC with different thicknesses $d$ of photoactive ZnTe layer. Curve 1 corresponds to the case when $d \geq w+L_{d}\left(L_{d}\right.$ is the diffusion length for minority charge carriers). One can see that photosensitivity is practically absent in the UV spectral region because of light and recombination losses of photocurrent in the thick ZnTe layer. Curve 2 


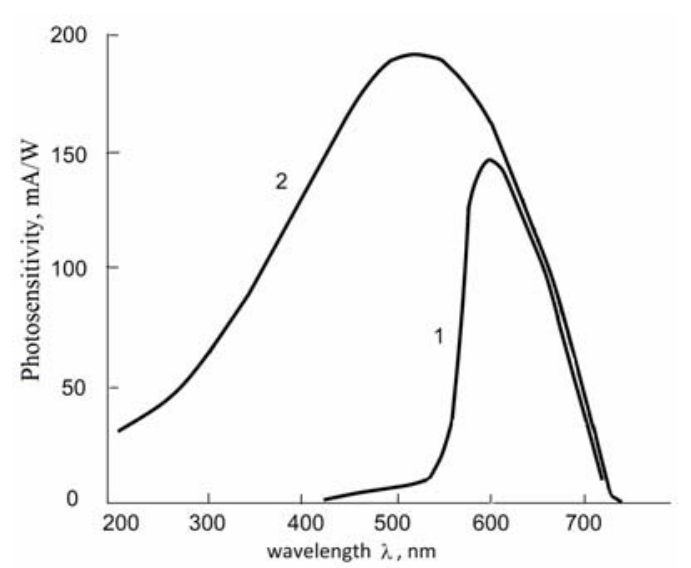

Fig. 3. Photosensitivity spectra for $p-\mathrm{Cu}_{1.8} \mathrm{~S} / p-\mathrm{ZnTe} / n$-CdSe PEC: $1-d \geq w+L_{\mathrm{d}} ; 2-d \leq w(d$ is the ZnTe layer thickness, $w$ - SCR width in ZnTe, $L_{d}$ - diffusion length for minority charge carriers).

corresponds to spectral sensitivity of PEC with optimal ZnTe layer thickness: $d \leq w$. Therefore, the studied sensors do not rank below the well known analogs [5] in absolute values of photocurrent in the UV spectral region.

\section{Conclusion}

Our investigations showed that $p$-ZnTe $/ n$-CdSe heterojunction pair, lattice parameters of which differ by less than $1 \%$, are promising for making sensors of UV radiation in the case of the surface-barrier structure version. The advantage of $p-\mathrm{Cu}_{1.8} \mathrm{~S} / p-\mathrm{ZnTe} / n$-CdSe over the known surface-barrier structures of Schottky barrier type is that the former ones have no parasite photosensitivity in the near IR spectral region that is related to emission of photoelectrons from metal to semiconductor. Our investigations of the crystal structure inherent to polycrystalline ZnTe layers grown on different substrates showed that it is impossible to achieve ideal lattice matching for the $p-\mathrm{ZnTe} / n-\mathrm{CdSe}$ heterojunction pair by growing $(\mathrm{ZnTe})_{x}(\mathrm{CdSe})_{1-x}$ transition layers. It seems that one should use $\mathrm{CdSe}_{x} \mathrm{~S}_{1-x}$ solid solution to achieve ideal lattice matching and consequently increase UV sensor photosensitivity.

\section{References}

1. Yu.N. Bobrenko, S.Yu. Pavelets, A.M. Pavelets, The efficient photoelectric converters of UV radiation with ZnS-based graded-gap layers // Semiconductors, 43, p. 801-805 (2009).

2. Yu.N. Bobrenko, S.Yu. Pavelets, A.M. Pavelets, N.V. Yaroshenko, Photoelectric converters with graded-gap layers based on $\mathrm{ZnSe} / /$ Semiconductors, 47(10), p. 1372-1375 (2013).

3. Yu.N. Bobrenko, S.Yu. Pavelets, A.M. Pavelets, M.P. Kiselyuk, N.V. Yaroshenko, Efficient photoelectric converters of ultraviolet radiation based on $\mathrm{ZnS}$ and $\mathrm{CdS}$ with low-resistivity surface layers // Semiconductors, 44(8), p. 1080-1083 (2010).

4. Yu.N. Bobrenko, A.M. Pavelets, S.Yu. Pavelets, V.M. Tkachenko, Short-wavelength photosensitivity of surface-barrier structures with degenerate semiconductor-semiconductor junctions // Tech. Phys. Lett. 20, p. 477-480 (1994).

5. T.V. Blank, Yu.A. Goldberg, Semiconductor photoelectric converters for the ultraviolet region of the spectrum // Semiconductors, 37(9), p. 999-1030 (2003), in Russian.

6. Physics of II-VI Compounds, Eds. A.N. Georgobiani, M.K. Sheinkman. Nauka, Moscow, 1986 (in Russian).

7. A.G. Milns, D.L. Feucht, Heterojunctions and Metal-Semiconductor Junctions. Academic Press, New York and London, 1972.

8. R.L. Anderson, Germanium-gallium arsenide heterojunctions // IBM J. Res. Develop. 4(3), p. 283-287 (1960).

9. Su-Huai Wei, A. Zunger, Calculated natural band offsets of all II-VI and III-V semiconductors: Chemical trends and the role of cation $d$ orbitals // Appl. Phys. Lett. 72(16), p. 2011-2013 (1998).

10. A.A. Toropov, S.V. Ivanov, Y.S. Park et al., Electroabsorption and laser generation in $\mathrm{ZnCdSe} / \mathrm{ZnSeS}$ quantum well diodes // Fizika, Tekhnika Poluprovod. 30(4), p. 656-669 (1996), in Russian.

11. K. Ando, H. Ishikura, Y. Fukunaga, T. Kubota, H. Maeta, T. Abe, H. Kasada, Highly efficient blue-ultraviolet photodetectors based on II-VI wide-bandgap compound semiconductors // phys. status solidi (b), 229(2), p. 1065-1071 (2002). 